\section{Lung ultrasound: a valid help in the differential diagnosis between pneumothorax and pulmonary blebs}

\author{
Francesca Sandionigi, \\ Francesca Cortellaro, Elisa Forni, \\ Daniele Coen \\ Emergency Department, Niguarda \\ Ca' Granda Hospital, Milano, Italy
}

\begin{abstract}
Spontaneous pneumothorax is a relatively common disease but its radiological diagnosis can be difficult because of the insufficient sensitivity of chest X-rays. This is even more so when bullous emphysema and acute pneumothorax co-exist. There is evidence that lung ultrasound is a valid instrument to detect a pneumothorax and a valid help in the differential diagnosis between pneumothorax and pulmonary blebs. We present a case which suggests the potential superiority of lung ultrasound on chest radiography in distinguishing free air (pneumothorax) from apical blebs, particularly when these are located in the posterior regions and may be easily confused with pneumothorax.
\end{abstract}

\section{Introduction}

Spontaneous pneumothorax (defined as the spontaneously occurring presence of air in the pleural space in patients without apparent underlying lung disease) is a relatively common disease primarily affecting young and otherwise healthy individuals. Pneumothorax typically occurs in tall, thin, male gender subjects; smoking is a risk factor. The spontaneous rupture of a subpleural bleb or of a bulla (usually at the lung apex) creates a communication between the alveolar spaces and the pleura.

An apical subpleural bleb is the most common cause of a primary spontaneous pneumothorax, while chronic obstructive pulmonary disease is responsible for most secondary spontaneous cases.

The presenting symptoms are sudden onset of dyspnea, pleuritic chest pain, cough and anxiety. Physical examination can be normal when the pneumothorax is small. Rapidly evolving hypotension, tachypnea, tachycardia, and cyanosis should raise the suspicion of a tension pneumothorax, which is extremely rare among primary forms. ${ }^{1}$

Chest X-rays studies classically show a white line (pleura) outlining a distinct area of black pleural space where lung markings are absent. It can be difficult to differentiate bullous emphysema from acute pneumothorax by chest X-rays, especially when both entities coexist; further, the sensitivity of X-rays is particularly low when the amount of air in the pleural space is limited. Computed tomography (CT) is the most sensitive and specific modality for the detection of a pneumothorax and remains the reference standard. However, the use of this diagnostic technique has drawbacks in terms of costs, high dose of radiation, transport of patients and loss of time. Lung ultrasound is a valid alternative, as several studies demonstrate its accuracy in diagnosing a pneumothorax and defining its extension. ${ }^{2}$ We present a case where bedside ultrasound in the emergency department was crucial in the differential diagnosis between bullous disease and pneumothorax.

\section{Case Report}

A 21-year-old man was admitted to the emergency department of Niguarda Ca' Granda Hospital in Milan presenting a three-day-history of chest pain exacerbated by respiratory acts and associated with shortness of breath. He denied sputum production, cough and fever. A left pneumothorax had been treated surgically 3 months earlier.

Vital signs were in normal range as was pulse oxymetry; he had a shallow respiration with breath sounds clear and bilateral; cardiac and abdominal examinations were normal. He seemed very worried about a possible recurrence of pneumothorax, though the symptoms observed were mild. Electrocardiogram (ECG) provided evidence of sinus tachycardia (110 bpm) and no signs of acute ischemia or pericarditis. Chest X-ray showed a small area of hyperinflation with decreased lung markings at the right upper lobe, which was interpreted by the radiologist as a pneumothorax (Figure 1). Bedside ultrasound examination showed a normal sliding sign bilaterally excluding the presence of a pneumothorax (Figure 2). A chest CT performed 3 months earlier at another hospital helped with the diagnosis, showing a right posterior apical bleb that satisfactorily explained the current radiological images (Figure 3). Differential diagnosis of chest pain includes: myocardial ischemia, pericarditis, pleuritis, aortic dissection, pulmonary embolism, herpes zoster. All these diagnoses appeared less likely than pneumothorax or musculoskeletal pain, his young age, the characteristics of the pain (exacerbated by breathing), clinical examination, absence of risk factors and normal ECG and laboratory considered. The patient was then discharged with a
Correspondence: Daniele Coen, Emergency Department, Niguarda Ca' Granda Hospital, Piazza 0spedale Maggiore 3, 20162 Milano, Italy. Tel. +39.02 .64447433 - Fax: +39.02 .64442825 .

E-mail: daniele.coen@ospedaleniguarda.it

Key words: lung ultrasound, pneumothorax, pulmonary bleb.

Received for publication: 15 February 2013

Revision received: 5 May 2013.

Accepted for publication: 6 May 2013.

This work is licensed under a Creative Commons Attribution 3.0 License (by-nc 3.0).

(C) Copyright F. Sandionigi et al., 2013

Licensee PAGEPress, Italy

Emergency Care Journal 2013; 9:e3

doi:10.4081/ecj.2013.e3

diagnosis of non-specific thoracic pain and was prescribed analgesics. Called back in 7 days he reported well-being with complete resolution of symptoms.

\section{Discussion}

Blebs or cysts can be easily mistaken for a pneumothorax. The criteria for radiographic diagnosis of pneumothorax include: i) visualization of the visceral pleura separated from the chest wall with loss of lung markings laterally; ii) demonstration of a deep sulcus sign; iii) crisp definition of the hemidiaphragm; and iv) demonstration of a continuous diaphragm sign. ${ }^{3}$ When chest radiography cannot clearly provide the answer, a CT scan is recommended. The use of ultrasounds to diagnose pneumothorax was first described in a veterinary medical journal in 1986. The following year, Wernecke et al. described sonographic recognition of pneumothoraces in human subjects on the basis of absence of pleural sliding and comet tail artifacts. Since then, multiple case reports and several studies have explored the use of ultrasound in the diagnosis of pneumothorax. $^{4}$

Ultrasonographic examination of the lung and pleura can be perfomed using linear and convex probes of 3.5-7.5 MHz using intercostal spaces as an acoustic window.

Patients are best examined supine, as the free thoracic air associated with a pneumothorax will rise to the anterior chest wall at the location where the exam is performed. Bilateral pleural interfaces are examined at the second to fourth intercostal spaces anteriorly in the parasternal line and up to the midaxillary line to look for the lung point. ${ }^{3}$ In the supine patient, the sonographic technique 
consists of exploring the least gravitationally dependent areas progressing more laterally. ${ }^{5}$ The study is performed at bedside, without the need to transport the patient and may be repeated as often as clinically indicated.

The primary goal is to visualize normal sliding of the lung at the pleural line. Pleural sliding is the to-and-fro movement of the visceral pleura against the parietal pleura during respiration; the presence of air in the pleural cavity acts as a scatter of sound that prevents sono-

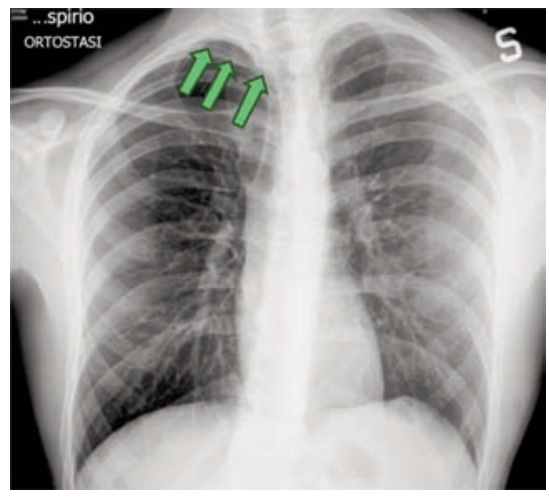

Figure 1. Chest radiography revealing a possible small apical right pneumothorax (arrows).

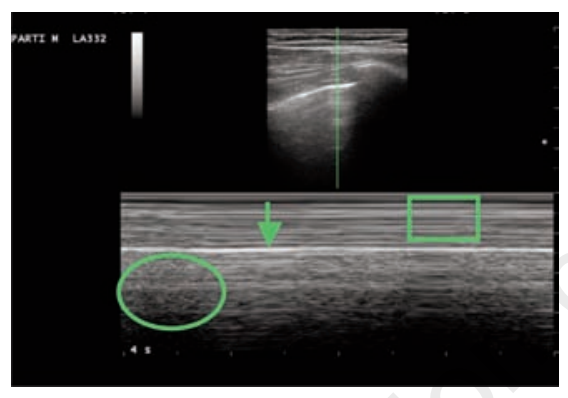

Figure 2. Motion-mode ultrasound image of lung revealing normal pleural sliding. The rectangle represents waves from superficial thoracic tissue; the vertical arrow indicates the pleural line; the ellipse shows sand from moving lung.

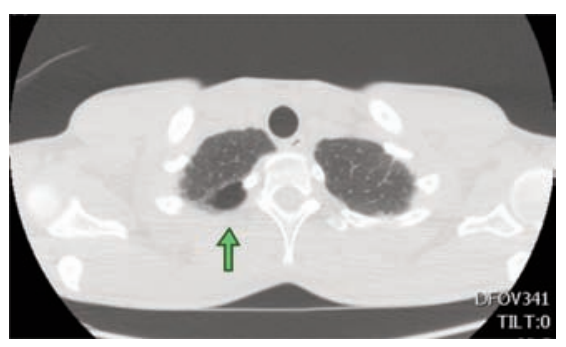

Figure 3. A previous pulmonary computed tomography with evidence of an apical right bleb (arrow). graphic visualization of visceral pleural movements, so the presence of pleural sliding excludes the diagnosis of pneumothorax. The abolition of sliding alone had a sensitivity of $100 \%$ and specificity of $78 \%$ for the diagnosis of occult pneumothorax; ${ }^{6}$ a study of Dulchavsky et al. reports a true negative rate of $100 \%$ for ruling out a pneumothorax when the sliding lung sign has been shown. ${ }^{7}$

The sliding sign may be shown in the motion (M)-mode: this image is called the seashore sign and is characterized by horizontal lines (waves) representing the static chest wall, and a scattered region (sand), formed by the dynamic artifacts beyond the pleural line during its normal movement (Figure 2), which would be absent in the presence of a pneumothorax. ${ }^{8}$

Lung pulse is sliding due to the transmission of heart beat movements to the pleural line: its presence, for the reasons above, excludes a pneumothorax.

B-lines are vertical hyperechoic artifacts that arise from the pleural line and extend to the lower edge of the image, moving with respiration, and erase the physiologic horizontal $A$-line artifacts seen in normal lungs. The presence of even a single B-line indicates the absence of pneumothorax with a negative predictive value of $100 \%$. $^{9}$

In case of absence of lung sliding, pulse and $B$ lines, the lung point sign should be detected. This is the visualization of lung sliding and absent lung sliding in the same intercostal space, representing the movement of the lung at the border of the pneumothorax; it is useful both to confirm the presence of a pneumotho$\operatorname{rax}$ (specificity 100\%) and to delineate the lateral margin of the air collection and thus its extension. ${ }^{10}$

The use of ultrasounds has contributed to a better recognition of occult pneumothorax with greater sensitivity and negative predictive value than chest radiography and with a similar specificity. Supine anteroposterior (AP) chest radiography has been reported to have very poor sensitivity in the detection of pneumothorax (36-75\%), while the sensitivity of lung ultrasound is higher (92-98\%) with similar specificity (both of 99-100\%); the accuracy of chest radiography and ultrasound is respectively 74 and $96 \%{ }^{2,3,6,7,11,12}$

In addition, lung ultrasound allows the detection of a pneumothorax within 2 to $4 \mathrm{~min}$ compared to the 20 to 30 min necessary for chest radiography. ${ }^{13}$

Thoracic ultrasonography has also been shown to be accurate in identifying the size and extension of a pneumothorax. ${ }^{2,14}$

This case reinforces the potential superiority of ultrasounds on chest radiography to distinguish between free air (pneumothorax) and apical blebs, particularly when located in the posterior regions where they determine the composition of a radiological image which may be easily confused with a pneumothorax. Echography is an operator-dependent technique, but its acquisition needs only a short period of training ${ }^{15,16}$ and findings of pleural disease are relatively simple to detect.

Lung echography has intrinsic limitations since it offers poor visualization of the mediastinum and is impaired by subcutaneous emphysema, obesity, pleural adhesions or any condition in which the pleural surfaces do not slide against each other with respiration. When a big bleb is located against the parietal pleura, sliding may be minimal because there may be little or no movement of the visceral pleura that covers the bleb, so that the M-mode pattern may resemble a pneumothorax. ${ }^{17}$ However, since there is no free air in the pleural space, comet tailing and the pulse sign are likely to be present. ${ }^{18}$

\section{Conclusions}

This case shows that lung ultrasound could be used in clinical settings when pneumothorax is in the differential diagnosis with pleural blebs, distinguishing free air in the pleural cavity (no B lines, absence of sliding, and presence of lung point).

In case of large apical blebs, lung sliding could be absent: the presence of $B$ lines and the absence of lung point support the diagnosis of blebs $v s$ pneumothorax.

This is just one more reason to implement this technique in emergency departments. We believe that thoracic ultrasound should become a widespread tool for emergency physicians in the near future.

\section{References}

1. Noppen M, De Keukeleire T. Pneumothorax. Respiration 2008;76:121-7.

2. Ball CG, Kirkpatrick AW, Feliciano DV. The occult pneumothorax: what have we learned? Can J Surg 2009;52:e173-9.

3. Rowan KR, Kirkpatrick AW, Liu D, et al. Traumatic pneumothorax detection with thoracic US: correlation with chest radiography and CT - initial experience. Radiology 2002;225:210-4.

4. Wilkerson RG, Stone MB. Sensitivity of bedside ultrasound and supine anteroposterior chest radiographs for the identification of pneumothorax after blunt trauma. Acad Emerg Med 2010;17:11-7.

5. Volpicelli G, Elbarbary M, Blaivas M, et al. International evidence-based recommendations for point-of-care lung ultrasound. Intens Care Med 2012;38:577-91. 
6. Lichtenstein DA, Mezière G, Lascols $\mathrm{N}$, et al. Ultrasound diagnosis of occult pneumothorax. Crit Care Med 2005;33:1231-8.

7. Dulchavsky SA, Schwartz KL, Kirkpatrick AW, et al. Prospective evaluation of thoracic ultrasound in the detection of pneumothorax. J Trauma 2001;50:201-5.

8. Soldati G, Testa A, Silva FR, et al. Chest ultrasonography in lung contusion. Chest 2006;130:533-8.

9. Lichtenstein D, Mezière G, Biderman P, et al. The comet-tail artifact: an ultrasound sign ruling out pneumothorax. Intens Care Med 1999;25:383-8.

10. Lichtenstein D, Mezière G, Biderman P, et al. The "lung point": an ultrasound sign specific to pneumothorax. Intens Care
Med 2000;26:1434-40.

11. Soldati G, Sher S. Bedside lung ultrasound in critical care practice. Minerva Anestesiol 2009;75:509-17.

12. Agricola E, Arbelot C, Blaivas M, et al. Ultrasound performs better than radiographs. Thorax 2011;66:828-9.

13. Bouhemad B, Zhang M, Lu Q, et al. Clinical review: bedside lung ultrasound in critical care practice. Crit Care 2007;11:205.

14. Blaivas M, Lyon M, Duggal S. A prospective comparison of supine chest radiography and bedside ultrasound for the diagnosis of traumatic pneumothorax. Acad Emerg Med 2005;12:844-9.

15. Soldati G, Testa A, Sher S, et al. Occult traumatic pneumothorax. Diagnostic accuracy of lung ultrasonography in the emergency department. Chest 2008;133: 204-11.

16. Sartori S, Tombesi P. Emerging roles for transthoracic ultrasonography in pleuropulmonary pathology. World J Radiol 2010;2:83-90.

17. Campione A, Luzzi L, Gorla A, et al. About ultrasound diagnosis of pulmonary bullae vs. pneumothorax. J Emerg Med 2010;38: 384-5.

18. Simon BC, Paolinetti L. Two cases where bedside ultrasound was able to distinguish pulmonary bleb from pneumothorax. J Emerg Med 2005;29:201-5. 